\title{
Per una revisione del concetto di "modello costituzionale": un'analisi della Costituzione Bavarese del 1818
}

\author{
Markus J. Prutsch
}

\section{Introduzione}

La Rivoluzione Francese, che si può considerare un fenomeno paneuropeo, ebbe effetti duraturi anche dopo la diminuzione della foga rivoluzionaria, nella maggior parte degli Stati europei, tutti costretti, seppure in forme e gradi diversi, ad affrontare le sfide del retaggio della Rivoluzione in seguito al crollo dell'ordine napoleonico. La sfida principale dell'epoca fu forse quella di riconciliare la rivendicazione del diritto da parte dei monarchi europei a preservare la propria sovranità con le aspettative delle società postrivoluzionarie concentrate su uno stato costituzionale e sulla salvaguardia del rinnovamento politico conseguente alla Rivoluzione e al Regime napoleonico. L'approccio spagnolo al rigido neoassolutismo non era più considerato soddisfacente come risposta a lungo termine. ${ }^{1}$ Al contrario, l'esempio della Restaurazione francese sotto Luigi XVIII, che ambiva ad ottenere una riconciliazione duratura mediante l'offerta di garanzie costituzionali, sembrava una soluzione più appropriata e ragionevole.

Non c'è quindi da sorprendersi se la Restaurazione dei Borboni nel 1814 fu, come lo era stata la stessa Rivoluzione, un atto di rilevanza europea; un evento che poteva essere la chiave per superare definitivamente l'era rivoluzionaria. La Charte constitutionnelle ("Carta Costituzionale") giocò un ruolo essenziale in questo contesto: fu la pietra miliare del nuovo regime e offrì soluzioni plausibili per la riconciliazione delle diverse aspirazioni dei governanti e delle società postrivoluzionarie. ${ }^{2}$ Il nuovo sistema monarchico costituzionale riconosceva nel sovrano il potere politico dominante, dichiarandolo solo e unico detentore del pouvoir constituant; contemporaneamente, però, ne limitava il potere mediante una costituzione scritta, che fissava le libertà civili e permetteva ai cittadini di prendere parte al processo politico e, soprattutto, a quello legislativo. Per questo motivo il "costituzionalismo monarchico" della Charte francese, che si potrebbe giustamente definire constitutional monarchism, ${ }^{3}$ è stato spesso

\footnotetext{
${ }^{1}$ Nella sua relazione finale sul Congresso di Vienna, Talleyrand descrisse la delusione unanime dei poteri europei per il modo in cui Ferdinando VII si era reimpadronito della corona di Spagna, nel 1814. Si veda Rapport fait au Roi pendant son voyage de Gand à Paris (giugno 1815), in Correspondance inédite du Prince de Talleyrand et du Roi Louis XVIII. Pendant le Congrès de Vienne, ed. G. Pallain, Paris et al., E. Plon et al., 1881, pp. 436-484, in particolare p. 474.

${ }^{2}$ Charte constitutionnelle (4 giugno 1814), in Bulletin des lois du Royaume de France Vol. 1 No. 133, pp. 197-207.

${ }^{3}$ Si confronti con E.N. Anderson e P.R. Anderson, Political Institutions and Social Change in Continental Europe in the Nineteenth Century, Berkeley, University of California Press, 1967, p. 39f., p. 78f. L'uso del termine "costituzionalismo monarchico" ha una lunga tradizione nella legge costituzionale tedesca (monarchischer Konstitutionalismus), soprattutto come sinonimo dell'effettivo sviluppo costituzionale della Germania e, in particolare, della Prussia, durante il diciannovesimo secolo. Si veda, ad esempio, O.
} 
considerato un modello per l'Europa postnapoleonica, e, in particolare, per la Germania meridionale.

Tuttavia, se la Costituzione fu un potenziale "modello" per l'Europa, bisogna chiedersi che cosa tale modello significasse in termini pratici, e in che grado la Charte francese potesse essere essenzialmente "trasferita" e "recepita". Effettivamente, la Charte non era, al tempo, l'unico "modello" plausibile, bensì uno di molti. Quando i Borboni tornarono al potere nel 1814, trovarono una situazione politica, socioeconomica e culturale unica; analogamente, la Francia era stata sconfitta a livello militare e l'economia era al collasso. Il motivo alla base del cambiamento del sistema politico esistente era soprattutto legato al fatto che Napoleone aveva screditato il regime rifiutando di firmare la pace, nonostante i ripetuti tentativi degli Alleati, e, così facendo, aveva oberato le spalle del Paese di un peso intollerabile. Ma il regime della Restaurazione doveva anche confrontarsi con una società lacerata dalla diffidenza reciproca: l'esempio più ovvio riguardava coloro che cercavano di ricostituire l'ordine dell'Ancien Régime e coloro che promuovevano una società basata sui principi della Rivoluzione. Inoltre, visto che la storia delle costituzioni scritte in Francia era relativamente lunga e piena di eventi, qualsiasi nuovo progetto costituzionale, nonché qualsiasi proposta di Luigi XVIII, sarebbero stati automaticamente paragonati a quelle precedenti.

Questi fattori non erano presenti, perlomeno non così fortemente, nella Germania meridionale. Ma questi Stati avevano le proprie sfide da affrontare, soprattutto il consolidamento politico e finanziario, ${ }^{4}$ e l'adattamento del regime assolutistico esistente alle necessità più immediate. Si potrebbe quindi supporre che il "modello" costituzionale francese non si potesse trasferire direttamente alla Germania meridionale. Connesso all'ipotesi della presenza di strutture differenti è il fatto che gli Stati della Germania meridionale si erano fatti un'esperienza precostituzionale, e avevano una tradizione rappresentativa che doveva essere tenuta presente, specialmente per quanto riguardava il coinvolgimento nel nuovo sistema di quelli che erano stati i Landstände (“Assemblea degli Stati”). Questa "eredità" reale o immaginaria sarebbe diventata sempre più importante in un'atmosfera di crescente nazionalismo e riserve sempre maggiori nei confronti degli influssi stranieri.

Il presente articolo si propone di evidenziare, all'interno di questo contesto, il "modelloruolo" della Charte constitutionnelle in quanto prototipo del costituzionalismo monarchico, con particolare attenzione ad un esempio specifico, quello della Costituzione Bavarese del 1818,

Hintze, Das monarchische Prinzip und konstitutionelle Verfassung, in «Preußische Jahrbücher» 144 (3), 1911, pp. 381-412. Tuttavia, il termine caratterizza in modo appropriato anche la situazione costituzionale francese, nonché quella di altri Stati europei, dopo il 1814.

${ }^{4}$ In particolare, l'amministrazione del debito pubblico moderno dev'essere considerata una parte necessaria della politica riformista. In merito a tale relazione, specialmente in Baviera e nel Baden, si veda H.-P. Ullmann, Staatsschulden und Reformpolitik. Die Entstehung moderner öffentlicher Schulden in Bayern und Baden 1780-1820, Göttingen, Vandenhoeck \& Ruprecht, 1986. 
generalmente considerata come diretto "successore" della Charte francese del 1814. ${ }^{5}$ Più concretamente, la questione alla base di questo studio è la seguente: in quale misura la Costituzione Bavarese, la prima Costituzione postnapoleonica di uno Stato territoriale tedesco, fu influenzata dalla Charte francese o da altri possibili modelli costituzionali? Si può giustamente parlare di una percezione e di un'assimilazione conscia di uno o più modelli esistenti? La risposta a questa domanda potrebbe portare a nuove intuizioni riguardo alla natura e ai limiti del trasferimento costituzionale in generale, contribuendo così a migliorare la nostra conoscenza dei processi di scambio legale transnazionali.

\section{La Costituzione Bavarese del 1818: "modelli" e paralleli}

Il testo della nuova Verfassungs-Urkunde des Königreichs Baiern, promulgata il 26 maggio 1818, dopo quattro anni di delibere costituzionali all'interno del governo, fu suddiviso in un preambolo, nel quale venivano reiterate le ragioni alla base dell'emanazione della costituzione nonché le sue principali garanzie, e in dieci "titoli", costituiti in tutto da 122 paragrafi. ${ }^{6}$ Sebbene la struttura non richiamasse immediatamente quella di altri testi, vi erano ovvi paralleli tra la Costituzione Bavarese e la Charte constitutionnelle in particolare. Come la Charte francese, la Costituzione Bavarese possedeva la caratteristica generale di un atto volontario di autolimitazione da parte del monarca, e ripudiava fortemente, quindi, qualsiasi forma di sovranità popolare. Allo stesso tempo, la Costituzione rivendicava il suo essere il prodotto di

\footnotetext{
${ }^{5}$ Tale stima può considerarsi ormai condivisa, specialmente nella letteratura tedesca recente. Si veda, ad esempio, M. Kirsch, Monarch und Parlament im 19. Jahrbundert. Der monarchische Konstitutionalismus als europäischer Verfassungstyp - Frankereich im Vergleich, Göttingen, Vandenhoeck \& Ruprecht, 1999, in particolare pp. 322-329; E. Fehrenbach, Bürokratische Reform und gesellschaftlicher Wandel. Die badische Verfassung von 1818, in E.O. Bräunche e T. Schnabel (Eds.), Die Badische Verfassung von 1818. Südwestdentschland anf dem Weg zur Demokratie, Ubstadt-Weiher, Verlag Regionalkultur, 1996, pp. 13-24, explicitly p. 13; C. Schulze, Frübkonstitutionalismus in Deutschland, Baden-Baden, Nomos, 2002, p. 63; H. Brandt, Europa 1815-1850. Reaktion - Konstitution - Revolution, Stuttgart, Kohlhammer, 2002, p. 142; H. Brandt, Der lange Weg in die demokratische Moderne. Deutsche Verfassungsgeschichte von 1800 bis 1945, Darmstadt, Wissenschaftliche Buchgesellschaft, 1998, p. 68f.

"Verfassungs-Urkunde des Königreichs Baiern (26 maggio 1818; d'ora in poi "VU"). La versione ufficiale è pubblicata in Gesetzblatt für das Königreich Bayern (6 giugno 1818), colonna 101ff. Altre versioni sono state pubblicate, ad esempio, in Dokumente zur deutschen Verfassungsgeschichte. 1, Deutsche Verfassungsdokumente 1803-1850, ed. E.R. Huber, Stuttgart et al., W. Kohlhammer, 1978 [1961], No. 53, pp. 155-171, e Bayerische Verfassungsurkunden. Dokumentation zur bayerischen Verfassungsgeschichte, ed. A. Wenzel, Stamsried, E. Vögel, 2002 [1990], pp. 23-41. Per una traduzione inglese si veda, ad esempio, British and Foreign State Papers. 1817-1818. Volume 5, London, J. Ridgway and Sons, 1837, pp. 1055-1076. La Costituzione Bavarese fu la settima ad essere realizzata in Germania dopo il 1814, ma la prima ad essere promulgata in uno Stato tedesco piuttosto esteso. Il 26 maggio 1818, assieme al testo costituzionale in quanto tale, furono promulgati dieci editti complementari (Beylagen), che contenevano, tra le altre cose, regolamentazioni dettagliate sulla libertà di stampa (terzo editto), sui privilegi e la posizione della nobiltà (editti dal quarto al sesto), e sul corpo rappresentativo (decimo editto). Per una versione annotata degli editti si veda Die Verfassungs-Urkunde des Königreichs Bayern mit den bierauf bezüglichen Gesetzen und sonstigen Bestimmungen. Neuabdruck des amtlichen Textes mit Hinweisen auf die bisher erfolgten Änderungen für den Gebrauch des beiden Kammern des Landtages herausgegeben vom Landtagsarchivariat, München, E. Mühlthaler, 1909 [1899], pp. 65-227.
} 
una pura sovranità monarchica, dichiarandosi il frutto «del nostro [del Re] libero arbitrio». ${ }^{7}$ Ciò era coerente con l'allontanamento della nuova Costituzione Bavarese del 1818 dalla terminologia utilizzata in quella precedente, emanata nel 1808, a partire dal nome stesso: infatti, fu battezzata non più Konstitution, ma Verfassungs-Urkunde, così da evidenziare la rottura con il costituzionalismo rivoluzionario e napoleonico ${ }^{8}$ Altre importanti caratteristiche furono anch'esse "prese a prestito" dalla Carta Costituzionale francese: ad esempio il sistema bicamerale con una prima camera influente, il diritto esclusivo d'iniziativa legislativa da parte del monarca, e il diritto del sovrano di dissolvere e riunire il Parlamento.

Un'attenta comparazione testuale rivela una somiglianza presente non solo nelle principali caratteristiche delle due costituzioni, ma anche nella formulazione di molti articoli. Questo vale specialmente per il "principio monarchico", che divenne il $\S 1$ del secondo titolo della Costituzione Bavarese. «Il re è il capo supremo dello stato: egli riunisce nella sua persona tutti i diritti del supremo potere, e li esercita dietro le determinazioni da lui medesimo stabilite con questo atto costituzionale. La sua persona è sacra e inviolabile». L'adozione di questo articolo era merito di Georg Friedrich von Zentner (1752-1835), sicuramente la personalità più importante e influente all'interno della commissione cui era stata affidata la stesura della nuova Costituzione Bavarese, e il quale Metternich avrebbe più tardi definito «padre della Costituzione Bavarese». ${ }^{9}$ Per Zentner, la Corona doveva rimanere il centro dell'autorità politica, e le ambizioni parlamentari atte a sfidare la sovranità monarchica dovevano essere contrastate preventivamente. Gli articoli della Charte constitutionnelle francese che miravano a enfatizzare il potere sovrano del re all'interno del sistema costituzionale sembravano perfetti per questo scopo: «Art. 13. La personne du roi est inviolable et sacrée»; «Art. 14. Le roi est le chef suprême de l'Etat (...)» Di conseguenza, Zentner aveva raccomandato con successo l'adozione degli articoli francesi in una mozione del 15 aprile 1818, così da «esprimere più chiaramente» il

\footnotetext{
7 Dal preambolo della Costituzione. In accordo con tale argomentazione paternalistica, anche il preambolo poneva l'accento sulla «perseveranza dei nostri sforzi per far progredire il benessere dei nostri popoli», sulla «nostra volontà altrettanto libera che incrollabile», e sui «paterni sentimenti» del monarca. Il testo si concludeva come segue: «Ecco, o Bavaresi, i principali caratteri di questa costituzione che Noi vi diamo di nostra volontà libera e piena. Cosiffatti sono i principii di un re il quale non vuole andar debitore della felicità del suo cuore e della gloria del suo trono, fuorché alla felicità della patria e all'amore del suo popolo». Nel presente saggio, gli articoli della Costituzione Bavarese sono presentati nella traduzione italiana, tratta da Raccolta di tutte le Costituzioni antiche e moderne, 2 vols., Torino, Tipografia Cassone, 1848, vol. 1., e disponibile anche online nel sito: http://www.dircost.unito.it/cs/pdf/18180526 germaniaCostituzioneBaviera ita.pdf, mentre quelli della Charte nel testo originale in francese.

${ }^{8}$ La volontà di evitare qualsiasi termine di stampo "rivoluzionario" e/o "napoleonico" traspare dal fatto che il preambolo della Verfassungs-Urkunde si riferisce alla Costituzione del 1808 definendola non tanto Konstitution quanto Verfassung. Analogamente, l'assemblea parlamentare del 1808 non viene definita National-Repräsentation, ma ständische Versammlung.

${ }^{9}$ Lettera di Metternich a Gentz, 30 giugno 1824, in Aus Metternich's nachgelassenen Papieren. Dritter Band, ed. R.C.L. Fürst von Metternich-Winneburg, Wien, Braumüller, 1882, p. 134.
} 
«carattere della monarchia». ${ }^{10}$ Altri paralleli testuali sono quelli che riguardano la riunione simultanea delle due camere, ${ }^{11}$ il ruolo predominante della Camera Bassa in materia di legislazione fiscale, ${ }^{12}$ il diritto del Parlamento a presentare petizioni legislative, ${ }^{13}$ la procedura da seguire in caso di dissoluzione delle Camere, ${ }^{14}$ l'immunità dei parlamentari, ${ }^{15}$ il sanzionamento e la proclamazione dei progetti di legge, ${ }^{16}$ e l'organizzazione del potere giudiziario. ${ }^{17}$ Anche nell'elenco dei diritti fondamentali delle due costituzioni è presente un numero di analogie letterali, ed è probabile che i regolamenti della Costituzione Bavarese secondo cui: «Nessuno può sottrarsi al suo giudice ordinario»; «Nessuno può essere arrestato né perseguitato se non dopo le forme prescritte dalla legge»; e «Nessuno può essere costretto a cedere la sua proprietà per l'uso pubblico, se non dopo una decisione del consiglio di stato riunito e dopo esserne stato preventivamente reso indenne (...)» (IV. § 8) fossero basati direttamente sulla Charte francese: «Nul ne pourra être distrait de ses juges naturels» (Art. 62); «(...) personne ne pouvant être poursuivi ni arrêté que dans les cas prévus par la loi, et dans la forme qu'elle prescrit» (Art. 4) ; «L'Etat peut exiger le sacrifice d'une propriété, pour cause d'intérêt public légalement constaté, mais avec une indemnité préalable» (Art. 10). La garanzia dell'accesso illimitato da parte di tutti i cittadini a qualsiasi ufficio pubblico è anch'essa

${ }^{10}$ Bayerisches Hauptstaatsarchiv Staatsrat 1656. Un paragone testuale tra la Costituzione Bavarese, la Charte francese e altri testi costituzionali dell'epoca si trova in K. Usée, Der Einfluß der franqösischen Verfassungen auf die deutschen Verfassungsurkunden der Jahre 1806-1820, Dissertation, Universität Greifswald, 1910, pp. 58-79. Si veda anche J. von Hazzi, Ueber die Standpuncte der baierischen Verfassungs-Urkeunde von 1818 in Beziehung anderer Constitutionen, München, J. Lindauer, 1819. Per quanto riguarda la Charte francese si veda R. Oeschey, Die bayerische Verfassungsurkunde vom 26. Mai 1818 und die Charte Ludwigs XVIII. vom 4. Juni 1814. Ein Beitrag zur Lehre vom monarchischen Prinzip. Unter Berücksichtigung der bayerischen Konstitution vom 1. Mai 1808 und deren Vorbildes, der westfälischen Verfassung vom 15. November 1807, München, C.H. Beck, 1914.

${ }^{11}$ VU VI. \16: «La sessione della camera dei senatori [Kammer der Reichsräte] si apre e si chiude nel tempo medesimo che quella dei deputati.» Charte Art. 25: «Elle [la Chambre des pairs; N.d.A.] est convoquée par le roi en même temps que la Chambre des députés des départements. La session de l'une commence et finit en même temps que celle de l'autre.»

12 VU VI. \ 18: «La proposizione della tassa si fa anzitutto nella camera dei deputati, ed è quindi rimandata da questi alla camera dei senatori.» Charte Art. 47: «La Chambre des députés reçoit toutes les propositions d'impôts; ce n'est qu'après que ces propositions ont été admises, qu'elles peuvent être portées à la Chambre des pairs.»

${ }^{13}$ VU VII. \19: «Gli stati generali hanno il diritto di sommettere al re i loro voti e le loro proposizioni in una forma conveniente, per rapporto a tutti gli oggetti compresi nella sfera della loro azione.» Charte Art. 19: «Les chambres ont la faculté de supplier le roi de proposer une loi sur quelque objet que ce soit, et d'indiquer ce qu'il leur paraît convenable que la loi contienne.»

14 VU VII. \23: «In quest'ultimo caso [cioè se il Parlamento viene dissolto, N.d.A.], una nuova elezione della camera dei deputati avrà luogo tutto al più dopo tre mesi.» Charte Art. 50: «(...) dans ce cas, il doit en convoquer une nouvelle dans le délai de trois mois.»

15 VU VII. \27: «Nessun membro è responsabile dell'opinione che avrà esternato nella seduta, se non sia davanti alla camera stessa e conforme alle regole della seduta.» Charte Art. 52: «Aucun membre de la Chambre ne peut, pendant la durée de la session, être poursuivi ni arrêté en matière criminelle, sauf le cas de flagrant délit, qu'après que la Chambre a permis sa poursuite.»

16 VU VII. \30: «Il re solo sanziona le leggi e le promulga (...)» Charte Art. 22: «Le roi seul sanctionne et promulgue les lois.»

17 VU VIII. \1: «La giustizia emana dal re.» Charte Art. 57: «Toute justice émane du roi.» 
formulata in modo identico: «Qualunque bavarese può indistintamente ottenere tutte le cariche civili, militari ed ecclesiastiche, come pure i benefizi» (IV. § 5); «Ils [les Français; N.d.A.] sont tous également admissibles aux emplois civils et militaires» (Art. 3).

Date queste evidenti somiglianze, non sorprende la frequente ipotesi, nella relativa letteratura, che la Costituzione Bavarese del 1818 fosse «essenzialmente basata sulla Carta Costituzionale di Luigi XVIII». ${ }^{18}$ Tuttavia, è necessario un po' di scetticismo di fronte a valutazioni tanto generali, soprattutto perché esistono paralleli testuali non solo con la Costituzione Francese del 1814, ma anche con altri "modelli" costituzionali, in particolare la bozza (reale) della Costituzione di Württemberg (1817), e la Costituzione Bavarese "napoleonica" del 1808. Si può sostenere che il testo della Costituzione di Württemberg fosse esso stesso basato sulla Charte constitutionnelle, e che quindi la ricezione della Charte in Baviera fosse stata solamente "mediata", il che sembra molto probabile sotto parecchi punti di vista. ${ }^{19}$ Ciò non spiega, tuttavia, l'utilizzo di articoli presi dalla Costituzione del 1808, che sono, in effetti, più numerosi di quelli derivati dalla Charte. In particolare i titoli II. (Del Re e della successione e della reggenza), IV. (Dei diritti e dei doveri generali), e IX. (Della costituzione militare), ricapitolano sostanzialmente gli articoli della Costituzione del $1808 .{ }^{20}$ Alcuni articoli dei due testi costituzionali avevano persino una formulazione identica: ad esempio, VU II. $\S 2$, e 1808 II. $\S 1$, «La corona è ereditaria nella linea maschile della casa reale, secondo l'ordine di primogenitura, e per collaterali maschi di ramo in ramo»; VU VIII. $§ 5$ e 1808 V. $§ 5$, «Il fisco reale, in ogni cosa che interessi i diritti di un particolare, litigherà davanti ai tribunali ordinarii». In termini puramente quantitativi, la Costituzione Bavarese del 1818 era dunque basata più sul suo predecessore nazionale del 1808 che su un modello straniero. Tuttavia, l'importanza dei "prestiti" testuali non dovrebbe essere sopravvalutata, poiché la loro utilità nel determinare la (non) importanza di un determinato modello costituzionale è limitata.

\section{Il "buon senso" legale e le peculiarità delle Costituzioni}

È chiaro che il significato e le implicazioni di contratti costituzionali simili, o persino identici, nella formulazione, possono differire in modo significativo da costituzione a costituzione, a

\footnotetext{
18 Usée, op. cit., p. 73.

${ }^{19}$ Le sezioni VI. (Von der Stände-Versammlung) e VII. (Von dem Wirkungskreise der Stände-Versammlung), in particolare, adottavano regolamenti provenienti dalla bozza della costituzione di Württemberg, presentata dal re alla Stände-Versammlung il 3 marzo 1817. Il testo si trova in K.H. Pölitz e F. Bülau, Die Verfassungen des teutschen Staatenbundes seit dem Jahre 1789 bis auf die neueste Zeit. Mit geschichtlichen Erläuterungen und Einleitungen. Band 1, Leipzig, F.A. Brockhaus, 1847, pp. 377-411. Si confrontino, ad esempio, VU VI 17 e Württ. 1817 Art. 275, VU VII \ 24 e Württ. 1817 Art. 300, VU VIII. \ 3 e Württ. 1817 Art. 160. È assai probabile che la formulazione del principio monarchico non si basasse direttamente sulla Charte francese, quanto sulla bozza della Costituzione di Württemberg (Art. 4).

20 Si confrontino, ad esempio, VU II. \4 e 1808 II. \2; VU II. \ 7 e 1808 II. \ 8; VU II. \ 10 e 1808

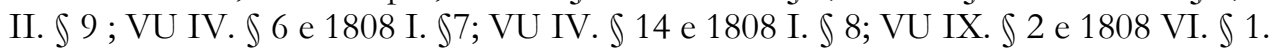


seconda del contesto culturale e politico e delle tradizioni giuridiche. Quindi, persino una dimostrazione esaustiva delle somiglianze tra costituzioni può avere solamente un valore limitato, se il contenuto e l'importanza delle norme non vengono analizzati all'interno dei rispettivi contesti. Analogamente, le somiglianze non sono necessariamente il risultato del desiderio di importare qualcosa di nuovo, ma possono essere dovute al fatto che una particolare costituzione funziona da "strumento supplementare" nel processo di redazione, dando così alle procedure (legali) esistenti, e a tutto il resto, un formato "costituzionale". Nelle deliberazioni della commissione costituzionale bavarese, tali riferimenti pragmatici ad altre costituzioni giocarono un ruolo importante, ed è proprio alla luce di questo fatto che bisogna interpretare le somiglianze tra gli articoli in difesa dei diritti di proprietà e dell'habeas corpus nella Charte e nella Costituzione Bavarese del 1818, ${ }^{21}$ cioè come il risultato di "incorporazioni tecniche", di per sé non del tutto innovative per la legge costituzionale bavarese. Inoltre, si può presumere che, nell'adottare un particolare sistema, come ad esempio la monarchia costituzionale, alcune impostazioni e istituzioni costituzionali venissero analogamente adottate, dato che le due cose sono legate a doppio filo. In altre parole: una volta presa la decisione di creare una monarchia costituzionale bavarese, ci si poteva attendere l'implementazione di una serie di istituzioni fondamentali che rappresentavano il nocciolo di questo tipo di costituzione. Tale osservazione potrebbe sembrare ovvia, ma viene spesso ignorata negli studi giuridici comparativi, che tendono a suggerire un trasferimento diretto tra due costituzioni, enfatizzando solamente come i casi in questione condividano le medesime istituzioni. In questo contesto, la Costituzione Bavarese del 1818 era tanto un'espressione scritta delle idee prevalenti relative al "governo costituzionale (ideale)", quanto lo era la Charte constitutionnelle francese; idee largamente costruite sulla percezione della legge costituzionale inglese.

Ciononostante, questo non significava che le costituzioni francese e bavarese dovessero essere quasi identiche. Una delle differenze più ovvie che distinguono il testo bavarese del 1818 dalla Charte è che il primo non garantiva ancora una piena eguaglianza secondo la legge pubblica, cosa che traspariva nell'esplicita differenziazione tra "Diritti e doveri generali" (Titolo IV.) e "Diritti speciali e privilegi" (Titolo V.). Una distinzione simile, che preservava essenzialmente un numero di diritti prerivoluzionari della nobiltà, ${ }^{22}$ non compare affatto nella Costituzione Francese del 1814, il cui primo articolo non lasciava dubbi sul fatto che «Les Français sont égaux devant la loi, quels que soient d'ailleurs leurs titres et leurs rangs». Il

\footnotetext{
21 Si confrontino VU IV. \ 8 e Charte Art. 4, 10 e 62.

22 Tra i privilegi garantiti alla nobiltà bavarese vi erano: V. \4: «1. il diritto esclusivo di poter esercitare una giustizia signorile; 2. il diritto di potere erigere i suoi beni immobili in fidecommessi; 3. l'esenzione dai tribunali di distretto nel civile e nel criminale; 4. il diritto detto del sigillo, nei limiti fissati dalle leggi sulle ipoteche; 5. il privilegio di fare entrare nella qualità di cadetti quelli dei loro figli che sarebbero colpiti dalla coscrizione.»
} 
mantenimento legale in Baviera di una "società a due classi" contraddiceva il paradigma di uguaglianza nel preambolo della Costituzione stessa, che prometteva «Dovere eguale di servire lo stato nell'onorata carriera delle armi», e «Eguaglianza nelle leggi e dei cittadini davanti ad esse», e fu perciò severamente criticata dai Liberali dell'epoca. ${ }^{23}$ Tuttavia, questa apparente incongruità fu comunque accettata dal governo bavarese, che considerava la continuazione dei privilegi legali come necessaria ad ancorare la nobiltà al nuovo ordine costituzionale, e a conquistare così un potente alleato nel periodo immediatamente successivo alla promulgazione della costituzione. La volontà di rafforzare l'elemento conservatore del sistema politico, appoggiando la nobiltà, traspare dalla particolare composizione delle due camere dell'Assemblea degli Stati Generali (Stände-Versammlung). Diversamente che in Francia, dove, sebbene gli aristocratici fossero sovrarappresentati nelle due camere parlamentari, il diritto di diventare membro di una delle due non era legato a un determinato privilegio di classe, ${ }^{24}$ la Costituzione Bavarese del 1818 non solo stabiliva che la prima camera (Kammer der ReichsRäthe) dovesse rappresentare quasi solamente la nobiltà terriera, ${ }^{25}$ ma riservava anche un ottavo dei seggi della seconda camera (Kammer der Abgeordneten) alla nobiltà (VI. § 9 a). ${ }^{26}$ La Costituzione Bavarese continuava a perpetuare, e non solo in questo senso, i rudimentali elementi delle forme tradizionali del governo corporativo, rimanendo, così, in forte contrasto coi moderni sistemi rappresentativi. L'intero procedimento elettivo della seconda camera era basato sulla rappresentanza proporzionale di classe, dato che le specifiche "classi" eleggevano un dato numero di rappresentanti per ciascun distretto elettorale governativo (Regierungsbezirk; VI. § 11). Oltre alla "Classe dei proprietari nobili" (VI. § 9 a), altri tre gruppi potevano nominare la maggior parte dei parlamentari (VI. § 9): «b) [la classe] degli ecclesiastici, così

\footnotetext{
${ }^{23}$ Si veda, ad esempio, Hazzi, op. cit., pp. 58-66. Nel fatto che alla nobiltà fosse permesso di essere esente dai tribunali civili e militari ordinari, Hazzi vede solo la conferma del feudalesimo tradizionale (ibid., p. 59). Non meno aspramente condanna gli altri privilegi garantiti dalla Costituzione.

${ }^{24}$ Per quanto riguarda la composizione della Chambre des pairs, l'Art. 27 della Charte dichiarava solamente che «La nomination des pairs de France appartient au roi. Leur nombre est illimité ; il peut en varier les dignités, les nommer à vie ou les rendre héréditaires, selon sa volonté.» Così, almeno in teoria, spettava al re nominare come membro della prima camera chiunque desiderasse. L'ammissione alla seconda camera era "aperta", perlomeno in quanto poteva ottenerla qualsiasi persona, indipendentemente dal proprio status sociale, che avesse oltre 40 anni e che avesse pagato una tassa annuale. Art 38: «Aucun député ne peut être admis dans la Chambre, s'il n'est âgé de quarante ans, et s'il ne paie une contribution directe de mille francs.»

25 Oltre al numero limitato di senatori avente il diritto di far parte della prima camera in quanto membri della dinastia reale (VI. \2 par. 1), come rappresentanti della nobiltà mediatizzata (VI. \2 par. 4) or ex officio (VI. $\ 2$ par. 2, 3, e 5) - ad esempio, i due arcivescovi - solo i proprietari terrieri aristocratici potevano essere nominati senatori per via ereditaria dal re (VI. \3). Il re aveva il diritto di nominare senatori a vita senza requisiti particolari (VI. $\int 2$ par. 6), ma «Il numero dei senatori a vita non può oltrepassare il terzo dei senatori ereditarii» (VI. \4).

${ }^{26} \mathrm{Il}$ numero dei rappresentanti della seconda camera non era fissato in termini assoluti, ma doveva essere determinato a seconda del numero di famiglie residenti nel regno prima di ogni elezione, di modo che vi fosse un deputato ogni 7000 famiglie (VI. S 8). Anche la Charte francese lasciava aperto il numero dei rappresentanti, ma utilizzava come base la popolazione dei dipartimenti.
} 
cattolici che protestanti, un ottavo essa pure; c) la classe delle città e dei borghi, un quarto; d) la classe degli altri proprietarii che non hanno giustizia signorile, metà del numero dei deputati». Le tre università bavaresi avevano anche loro un proprio rappresentante (VI. $\S 9 \mathrm{e}){ }^{27}$ Quindi, la maggior parte delle classi della popolazione erano rappresentati in un modo o nell'altro, ma in modo sproporzionato, dato che i proprietari terrieri e il clero erano significativamente sovrarappresentati. In questo senso, la Costituzione del 1818 era molto più "conservatrice" rispetto alla precedente del 1808, la quale non faceva tali distinzioni di classe, e il rifiuto della National-Repräsentation a favore della Stände-Versammlung non era in effetti solamente terminologico. ${ }^{28}$

Mentre la Costituzione Bavarese conservava alcuni elementi anacronistici del periodo prerivoluzionario - ad esempio, disuguaglianze legali e privilegi per determinati gruppi sociali, nonché il modo in cui era organizzata la seconda camera, evidente anche nel regolamento secondo cui l'Assemblea degli Stati Generali non dovesse mai diventare un'istituzione permanente e né si dovesse riunire a cadenza annuale ma triennale (VII. § 22) ${ }^{29}$ - il testo includeva anche delle caratteristiche innovative. Alcuni articoli andavano chiaramente oltre quelli della Charte francese, specialmente per quanto riguarda i diritti costituzionali delle camere. Mentre le competenze legislative generali erano piuttosto simili in Francia e in Baviera, e comprendevano soprattutto il diritto di decidere sulle proposte di legge presentate dalla Corona, incluse quelle relative alla legislazione sulla finanza e il bilancio, ${ }^{30}$ il diritto di petizione e la responsabilità dei funzionari statali erano più definiti nella Costituzione Bavarese rispetto alla Charte. In Baviera, il testo della costituzione prevedeva non solo il diritto delle camere a presentare petizioni legali in quanto prima forma di iniziativa legislativa parlamentare (VU VII. $\S 19$ e 20), come appunto nella Charte francese (Charte Art. 19-21), ma lo estendeva fino a

${ }^{27}$ Cioè le università di Landshut (prima Ingolstadt e più tardi Monaco), Würzburg ed Erlangen.

${ }^{28}$ Nella ventesima sessione della conferenza ministeriale, tenutasi il 9 aprile 1818, fu espressamente deciso che il «sistema rappresentativo» dovesse essere abbandonato in favore di quello «dell'Assemblea degli Stati Generali» (Bayerisches Hauptstaatsarchiv Staatsrat, 1656). Nella stessa sessione, fu accettata anche la proposta di Zentner, secondo cui si doveva modificare il nome dell'organo rappresentativo.

${ }^{29}$ Inoltre, si decise che le sessioni parlamentari non dovessero durare per più di due mesi, e che il compito principale del parlamento fosse quello di decidere riguardo alle leggi proposte dalla Corona e non quello di dare vita a dibattiti autonomi. Per quanto possibile, la Stände-Versammlung assomigliava più al Landtag tradizionale, che a un parlamento moderno.

${ }^{30} \mathrm{E}$ però notevole che la Charte riunisse in un unico articolo le competenze legislative delle camere, secondo cui ogni disegno di legge doveva passare attraverso due camere. Art. 18: «Toute la loi doit être discutée et votée librement par la majorité de chacune des deux chambres.» In Baviera, il testo costituzionale era più vago. Si veda, ad esempio, VII. \2: «Senza la deliberazione e l'assenso degli stati del regno, non si potrà dare alcuna legge generale che concerna la libertà delle persone o la prosperità dei sudditi dello stato, né recare alcun cambiamento ad una di quelle che già esistevano, né rivocarle, né darne una spiegazione autentica.» Inoltre, i diritti fiscali della Stände-Versammlung, come pure alcune importanti limitazioni di tali diritti in caso di «circostanze esterne e straordinarie» (VII. \7) e «bisogno straordinario e impreveduto» (VII. \8), sono elencati in dettaglio nella Costituzione Bavarese (si veda VII. \3-18). Nella Charte non sono presenti regolamentazioni di questo tipo. 
trasformarlo in un "diritto d'appello" formale per tutti i cittadini (VU VII. § 21): «Ciascun cittadino e ciascun comune può indirizzare all'assemblea degli Stati Generali o a ciascuna camera le sue lagnanze sulla violazione dei diritti costituzionali (...)» Quest'ampia interpretazione della petizione in quanto "appello" ma anche "reclamo", che non compare nella Charte francese, si fondava sul sistema costituzionale britannico, ed era accompagnata da una lettura più completa del "governo responsabile". ${ }^{31}$ Mentre la Charte prevedeva solo una responsabilità limitata da parte dei ministri reali (Art. 55 e 56), ${ }^{32}$ la Costituzione Bavarese del 1818 estendeva la responsabilità a tutti i funzionari statali nel caso di violazione della costituzione (X. § 4-6). ${ }^{33}$ In particolare, la novità stava nel fatto che questi significativi diritti parlamentari erano elencati in una sezione dedicata della Costituzione, intitolata "Della guarentigia della Costituzione" (Von der Gewähr der Verfassung). In linea con l'idea secondo cui la costituzione richiedeva una "garanzia" speciale, la Costituzione Bavarese - diversamente dalla Charte - chiariva anche la distinzione tra legislazione "normale" e legislazione "costituzionale", e istituiva una procedura specifica per alterare o emendare la Costituzione, la quale richiedeva una maggioranza legittima in entrambe le camere (X. § 7).

«Non possono essere fatti né cambiamenti né addizioni alla costituzione senza il consenso degli stati.. (...)

Per prendere una decisione in un affare di tanta importanza, è necessario [sic.] almeno la presenza dei tre quarti dei membri di ciascuna camera ed una maggioranza dei due terzi.» ${ }^{34}$

Tutto sommato, il testo della Costituzione Bavarese del 1818 assomiglia a una coperta a scacchi composta da vari elementi stranieri e nazionali, conservatori e liberali, tradizionali e

31 In Gran Bretagna, il Parlamento inoltrava tradizionalmente i reclami al governo sottoforma di "petizioni". Sul ruolo generale dell'Inghilterra in quanto modello per i dibattiti sulla Costituzione Bavarese tra la fine del diciottesimo e l'inizio del diciannovesimo secolo, si veda L. Lenk, Das Modell England in der bayerischen Verfassungsdiskussion zwischen 1770 und 1818, in Gesellschaft und Herrschaft. Forschungen zu sozial- und landesgeschichtlichen Problemen vornebmlich in Bayern. Eine Festgabe für Karl Bosl zum 60. Geburtstag, ed. R. van Dülmen, München, C.H. Beck, 1969, pp. 271-299.

32 Art. 55: «La Chambre des députés a le droit d'accuser les ministres, et de les traduire devant la Chambre des pairs qui seule a celui de les juger.»

Art. 56: «Ils ne peuvent être accusés que pour fait de trahison ou de concussion. Des lois particulières spécifieront cette nature de délits, et en détermineront la poursuite.»

${ }^{33} \mathrm{Si}$ veda, in particolare, $\ 4$ : «I ministri di stato del re e tutti i funzionarii pubblici sono responsabili del mantenimento della costituzione.» Diversamente dalla Gran Bretagna, però, il controllo parlamentare dell'organo esecutivo in termini di responsabilità politica rimase un obiettivo ancora lontano non solo in Francia, ma anche in Baviera.

34 Sul problema delle "gerarchie normative" definite e sulla preminenza della legge costituzionale, idea insita nel costituzionalismo americano della Germania del diciannovesimo secolo, si veda C.H. Schmidt, Vorrang der Verfassung und konstitutionelle Monarchie. Eine dogmengeschichtliche Untersuchung zum Problem der Normenhierarchie in den deutschen Staatsordnungen im frühen und mittleren 19. Jahrbundert (1818-1866), Berlin, Duncker \& Humblot, 2000. 
moderni. La Charte francese serviva come un'implicita ed esplicita "fonte di idee", ${ }^{35}$ ma allo stesso tempo i padri della Costituzione Bavarese avevano tenuto in considerazione anche altri punti di riferimento: i principi dei Landstände prerivoluzionari, la Costituzione del 1808, nonché il sistema costituzionale inglese. Il testo della Costituzione Bavarese conteneva analogamente le proprie novità, in particolare quando stabiliva strumenti tangibili per garantire la salvaguardia della Costituzione. Il carattere eterogeneo del testo potrebbe anche spiegare perché l'inviato francese a Monaco non vedesse all'epoca alcuna motivo di considerare la Costituzione Bavarese una conscia imitazione della Charte francese, come traspare dalle relazioni ufficiali inviate a Parigi. ${ }^{36}$

Tuttavia, ciò che condividevano la Costituzione Francese del 1814 e la Costituzione Bavarese del 1818, e che allo stesso tempo le separava dal sistema costituzionale inglese, era il principio della sovranità monarchica, che stravolgeva il concetto della separazione dei poteri di Montesquieu, dichiarando che la monarchia fosse un qualche tipo di "sovra-istituzione" costituzionale. Persino in questo senso pare che la Charte constitutionnelle non fosse necessariamente un modello "materiale" per l'adozione del principio monarchico, cioè una vera ispirazione nel processo costituzionale, che introduceva un elemento totalmente nuovo nella legge costituzionale bavarese. Era più che altro una formalità, che concedeva alla preesistente rivendicazione monarchica del pouvoir constituant una forma costituzionale chiara e tangibile. Persino in epoca napoleonica il proposito di concentrare tutta l'autorità statale nella Corona era diventato ovvio ed evidente nell'istituzione di un'amministrazione statale fortemente centralizzata e nell'abolizione delle forme governative corporative. Infine, la Charte francese conteneva anche la formulazione secondo cui la promessa di una costituzione poteva essere onorata senza abbandonare la rivendicazione di una sovranità assoluta; o, come scrisse Zentner: «Nel nostro stato, la sovranità è assoluta e riunita solamente nel reggente; solo il nostro presente sovrano - per rispetto verso le costituzioni corporative preesistenti e le garanzie conferite all'epoca della loro dissoluzione - ha limitato se stesso e i suoi successori nell'esercizio di

\footnotetext{
${ }^{35}$ Un esempio di tali adozioni "implicite" si ritrova nel modo in cui la Costituzione Bavarese del 1818 imitava la Charte nel presentare garanzie costituzionali per l'individuo non più in quanto diritti universali secondo la legge naturale, come nel periodo rivoluzionario, ma come semplici garanzie salvaguardate dal sovrano nei confronti dei cittadini, Ecco perché la Charte non fa riferimento ai Droit de l'homme, ma solamente al Droit public des Français.

${ }^{36}$ Sylvia Krauss esprime il proprio stupore per come il «Vorbildcharakter der französischen Charte für die bayerische Verfassung - ein Faktum», fu ignorato dall'inviato francese, La Garde (S. Krauss, Die politischen Beziehungen zwischen Bayern und Frankreich 1814/15-1840, München, C.H. Beck, 1987, p. 88). Si veda, ad esempio, le relazioni di La Garde in merito alla nuova Costituzione Bavarese, redatte il 13 aprile 1818, il 26 aprile 1818, il 10 maggio 1818, e il 27 maggio 1818, in Gesandtschaftsberichte aus München 1814-1848. Abteilung 1. Die Berichte der französischen Gesandten. Band 1, ed. A. Chroust, München, Biederstein, 1935, pp. 52-56.
} 
determinati diritti governativi; (...) il potere statale è dunque assoluto, ma limitato solamente dalle regole contenute nel Documento Costituzionale.» ${ }^{37}$

\section{Conclusione}

Dopo aver esaminato la Costituzione Bavarese, il trasferimento e la ricezione dei modelli costituzionali, appare chiaro come la Verfassungs-Urkunde continuasse la tradizione del costituzionalismo monarchico definito dalla Charte constitutionnelle, prevedendo un sistema bicamerale, ampio potere per la Corona e un ruolo limitato del Parlamento. Inoltre, vi sono forti somiglianze tra la Charte e la Costituzione Bavarese per quanto riguarda i diritti comuni e individuali. Il carattere e il ruolo della Charte nella progettazione della Costituzione Bavarese sono chiaramente evidenziati dalle analogie delle specifiche formulazioni, che non lasciano dubbi su come la Costituzione francese del 1814 avesse giocato un ruolo essenziale nella composizione delle costituzioni postnapoleoniche della Germania meridionale. In questo senso, si possono certamente avvalorare i risultati recenti derivati dalla ricerca storica comparativa.

Tuttavia, un'analisi più profonda pone dei limiti, sotto alcuni punti di vista, alla concezione della Charte in quanto unico e solo "modello" per le costituzioni della Germania meridionale. Il fatto è che la Charte fu un'importante, ma non unico, punto di riferimento per la Costituzione della Baviera e di altri Stati. Nel caso del documento bavarese, alcune delle formulazioni prese dalla Costituzione nazionale del 1808 e dal progetto reale della Costituzione di Württemberg del 1817 vennero frequentemente ripetute, spesso letteralmente. Inoltre, persino quando si possono determinare analogie tra la Costituzione francese del 1814 e la Costituzione Bavarese, ciò non implica un trasferimento diretto, né suggerisce un prestito esatto. I prestiti non erano sempre diretti, e in alcuni casi provenivano da una deviazione attraverso un altro Paese. In altri rispetti, la Costituzione Francese del 1814 era più uno specchio e un filtro delle pratiche e delle istituzioni costituzionali generali che un modello innovativo, e funzionava come una "entrata" per i topoi costituzionali predominanti dell'epoca, principalmente modellati sul sistema e le pratiche costituzionali britannici. Questo vale, ad esempio, per il sistema bicamerale. ${ }^{38}$ Analogamente, la Costituzione Bavarese modificava, riformulava e/o espandeva alcune regolamentazioni della Charte francese. Ma, nonostante l'impatto e gli effetti di questi prestiti,

\footnotetext{
${ }^{37}$ Dalla relazione di Zentner sulla Stände-Versammlung, pronunciata alla conferenza ministeriale del 7 luglio 1819. Citata in M. Doeberl, Ein Jahrhundert bayerischen Verfassungslebens, München, Lindauer, 1918, p. $50 f$. In linea con questa interpretazione, il preambolo della Costituzione Bavarese non considerava l'Assemblea degli Stati Generali come parte integrante della legislazione, ma solamente come una istituzione con «diritto di consigliare, di votare la legge».

${ }^{38} \mathrm{La}$ Charte ebbe anche il ruolo di "intermediario" per quanto riguarda i diritti fondamentali universali. Si veda W. von Rimscha, Die Grundrecbte im süddeutschen Konstitutionalismus. Zur Entstehung und Bedeutung der Grundrechtsartikel in den ersten Verfassungsurkunden von Bayern, Baden und Württemberg, Köln et al., C. Heymann, 1973, p. 43.
} 
il documento costituzionale bavarese conservava comunque un carattere originale e unico. La sua natura legata alla nazione la distingueva non solo dalla Charte, ma anche da altre costituzioni della Germania meridionale, come quella dello stato del Baden, nonostante vi fossero comunque molte somiglianze. ${ }^{39}$ Il modo in cui viene trattata l'eredità del Landstände ne è un chiaro esempio. La Costituzione Bavarese incorporava, o meglio "re-inventava", essenzialmente, forme di governo corporative, generando un edificio costituzionale alquanto ibrido, che ondeggiava tra i principi degli Altstände classici, il bisogno immediato di un governo rappresentativo, e la garanzia di una maggioranza schiacciante per la nobiltà terriera in entrambe le camere. ${ }^{40} \mathrm{Al}$ contrario, la Costituzione del Baden del 1818, promulgata poco dopo quella bavarese, adottava un sistema rappresentativo senza riserve.

In breve, si può affermare che la Costituzione Bavarese continuò la tradizione dei sistemi costituzionali monarchici iniziata dalla Charte contitutionnelle, la quale, sotto molti aspetti, fu d'esempio per la stesura di tutti i testi costituzionali della Germania meridionale. All'interno del principio del "costituzionalismo monarchico", tuttavia, c'era ampio spazio anche per l'individualità. Dunque, le costituzioni della Germania meridionale rappresentavano una complessa mescolanza di elementi esterni ed interni, tradizionali e innovativi. Il peso di ciascuno di questi componenti differiva per la specificità dei prerequisiti e il percorso del processo costituzionale. Differente allo stesso modo era la sfida che entrambi gli Stati si prefiggevano cercando di ravvivare i documenti costituzionali nell'ambito della politica pratica.

L'individualità di questi sistemi costituzionali mina le idee convenzionali secondo cui la Charte fu una traccia per le costituzioni della Germania meridionale, e fu semplicemente "copiata". Similmente, dimostra i limiti delle generalizzazioni legate alle recenti tipologie costituzionali. Tuttavia, mentre è ingannevole parlare troppo genericamente di una «epoca di ricezione delle leggi straniere», ${ }^{41}$ è non meno fuorviante enfatizzare oltremodo la specificità degli sviluppi costituzionali e dell'importanza della Sonderwege, espressa particolarmente nella letteratura tedesca precedente. Ciò significherebbe ignorare il fatto che la Charte era in ogni caso un'importante fonte di ispirazione per il costituzionalismo della Germania meridionale e, se non altro, perlomeno una "stazione intermedia" importante e una "porta d'entrata" formalizzata per le idee e le istituzioni costituzionali occidentali che avevano conquistato uno status universale.

\footnotetext{
39 In realtà, gli articoli della Costituzione Bavarese del 1818 vengono citati estensivamente nella Costituzione del Baden, sottolineando così la correlazione tra le costituzioni della Germania meridionale e la caratteristica del trasferimento in quanto processo di apprendimento.

${ }^{40}$ Joseph von Hazzi critica apertamente la sovrarappresentazione dell'aristocrazia nel suo studio sulla Costituzione Bavarese. Si veda Hazzi, op. cit., p. 77.

${ }^{41}$ C. Bornhak, Genealogie der Verfassungen, Breslau, M. \& H. Marcus, 1935, p. VI.
} 
Tutto sommato, si possono imparare alcune lezioni dai risultati di questo studio, per quanto riguarda la ricerca sul trasferimento e la ricezione interculturale: 1) le diverse condizioni e i prerequisiti strutturali non sono necessariamente un'argomentazione a sfavore del trasferimento e della ricezione di determinate idee e/o pratiche; 2) l'interpretazione tradizionale del "modello", ${ }^{42}$ ovvero del trasferimento da A a B, è problematica se non addirittura fuorviante, e quello che invece serve è una comprensione più flessibile e analitica della nozione di "modello"; 3) il trasferimento e la ricezione sono in effetti "processi" complessi, che operano a vari livelli e devono essere interpretati in quanto tali, e non semplicemente come un'unica fase autosufficiente; 4) è particolarmente difficile identificare quali prestiti sono atti consci e deliberati, e quali sono invece semplicemente atti meccanici o espressione del buon senso contemporaneo; 5) i resoconti sinottici che si occupano solamente delle diversità o delle somiglianze non sono sufficienti ai fini della comprensione del trasferimento e della ricezione; 6) è essenziale invece soffermarsi sui fattori individuali e sugli agenti del trasferimento e della ricezione, prendendo in considerazione il loro background politico, sociale ed educativo, ${ }^{43}$ in modo da ottenere un'idea più chiara di come funzionano i processi di trasferimento e ricezione; 7) poiché l'atto di ricezione e quello di trasferimento non sono solitamente descritti in dettaglio, bisogna accontentarsi di prove circostanziate, lasciando spazio ad interpretazioni ambigue. ${ }^{44}$ Date queste limitazioni di fondo, è evidente come 8) i concetti generalizzati formali forniscano solamente un aiuto limitato per lo studio del trasferimento e della ricezione, poiché non sono in grado di affrontare la complessità di questi fenomeni in quanto tali. Invece di tentare di creare una "teoria del trasferimento" generale, bisognerebbe sforzarsi di adottare approcci più "individuali", a seconda delle ricerche e delle fonti in questione.

$42 \mathrm{Si}$ veda, ad esempio, P. Pombeni, Political Models and Political Transfer in the Shaping of Europe, in «European Review of History» 12 (2), 2005, pp. 223-238.

${ }^{43}$ In questo contesto, i centri di scambio intellettuale come le università meritano maggiore attenzione rispetto a quanta ne abbiano ricevuta in passato. L'università di Göttingen, ad esempio, fu un centro molto importante, tra la fine del diciottesimo e l'inizio del diciannovesimo secolo, in cui studiarono esimi membri della commissione costituzionale bavarese, incluso Zentner, ma anche il Principe Ludwig.

${ }^{44}$ Mentre è facile individuare la "motivazione iniziale" delle parti coinvolte (ad esempio, l'essere pro o contro l'idea di una sovranità popolare) a partire da prove circostanziate, è molto più difficile comprendere particolari decisioni, come ad esempio se adottare o meno un determinato articolo o una determinata regola. 